07

\title{
Особенности оптического усиления в сильнолегированных $\mathrm{Al}_{x} \mathrm{Ga}_{1-x} \mathrm{~N}: \mathrm{Si}$-структурах
}

\author{
(ㄱ П.А. Бохан ${ }^{1}$, К.С. Журавлёв ${ }^{1}$, Д.Э. Закревский ${ }^{1,3, \uparrow, ~ Т . В . ~ М а л и н ~}{ }^{1}$, И.В. Осинных ${ }^{1,2}$, Н.В. Фатеев ${ }^{1,2}$ \\ ${ }^{1}$ Институт фризики полупроводников им. А.В. Ржанова СО РАН, Новосибирск, Россия \\ ${ }^{2}$ Новосибирский государственный университет, Новосибирск, Россия \\ ${ }^{3}$ Новосибирский государственный технический университет, Новосибирск, Россия \\ ๑ E-mail: zakrdm@isp.nsc.ru
}

Поступило в Редакцию 24 марта 2021 г.

В окончательной редакции 21 апреля 2021 г.

Принято к публикации 22 апреля 2021 г.

Экспериментально исследовано временно́е поведение интенсивностей люминесценции и стимулированного излучения в сильнолегированных структурах $\mathrm{Al}_{0.65} \mathrm{Ga}_{0.35} \mathrm{~N}$ и $\mathrm{Al}_{0.74} \mathrm{Ga}_{0.26} \mathrm{~N}$ при импульсном оптическом возбуждении. Результаты продемонстрировали, что временно́е затухание интенсивностей люминесценции и стимулированного излучения для различных длин волн излучаемого спектра и мощностей оптической накачки состоит по крайней мере из двух компонент: быстрой и медленной. Быстрые компоненты с экспоненциальным временны́м затуханием связаны с излучательной рекомбинацией неравновесных электронов на глубоких акцепторах, а медленные - с рекомбинацией донорно-акцепторных пар.

Ключевые слова: сильнолегированные структуры $\mathrm{Al}_{x} \mathrm{Ga}_{1-x} \mathrm{~N}$, люминесценция, стимулированная эмиссия, оптическое усиление.

DOI: 10.21883/PJTF.2021.14.51186.18782

Широкозонные (3.4-6.2 eV) структуры $\mathrm{Al}_{x} \mathrm{Ga}_{1-x} \mathrm{~N}$ являются перспективными материалами для создания источников света и лазерных диодов в видимой и УФ-областях спектра [1-3]. В структурах $\mathrm{Al}_{x} \mathrm{Ga}_{1-x} \mathrm{~N}$, легированных кремнием с концентрацией $n_{\mathrm{Si}}>10^{19} \mathrm{~cm}^{-3}$, присутствуют люминесцирующие дефекты, которые приводят к появлению внутризонных (внутри запрещенной зоны) излучательных переходов. Излучательные характеристики сильнолегированных структур $\mathrm{Al}_{x} \mathrm{Ga}_{1-x} \mathrm{~N}: \mathrm{Si}$ ранее были исследованы при возбуждении оптическим излучением [4]. Исследования продемонстрировали, что люминесценция структур $\mathrm{Al}_{x} \mathrm{Ga}_{1-x} \mathrm{~N}$ с $n_{\mathrm{Si}}>10^{20} \mathrm{~cm}^{-3}$ при $x>0.5$ имеет доминирующий широкополосный спектр $1.72-2.7 \mathrm{eV}(400-720 \mathrm{~nm})$. При оптической накачке квантовая эффективность люминесценции структур $\mathrm{Al}_{x} \mathrm{Ga}_{1-x} \mathrm{~N}: \mathrm{Si}$ с $x=0.65$ и 0.74 достигает 0.5 и 0.8 соответственно [4]. В работах [5,6] были измерены коэффициенты оптического усиления, превышающие $10^{3} \mathrm{~cm}^{-1}$, и продемонстрирована перестраиваемая лазерная генерация в структуре $\mathrm{Al}_{0.74} \mathrm{Ga}_{0.26} \mathrm{~N}$ при оптической накачке. Эти особенности делают сильнолегированные структуры $\mathrm{Al}_{x} \mathrm{Ga}_{1-x} \mathrm{~N}$ перспективными активными средами для получения лазерной генерации в видимом спектральном диапазоне, в том числе с большой перестройкой частоты излучения с единичного излучающего элемента, а также в качестве широкополосных светоизлучающих структур.

При возбуждении неравновесных носителей заряда в структурах $\mathrm{Al}_{x} \mathrm{Ga}_{1-x} \mathrm{~N}: \mathrm{Si}$ происходит одновременно несколько процессов, приводящих к излучательной релаксации энергии возбуждения. Целью настоящей рабо- ты является определение механизмов, ответственных за оптическое усиление.

Исследовались две структуры $\mathrm{Al}_{x} \mathrm{Ga}_{1-x} \mathrm{~N}: \mathrm{Si}$ толщиной $1.2 \mu \mathrm{m}$ с $x=0.65$ и 0.74 с буферным слоем AlN, выращенные методом молекулярно-лучевой эпитаксии на (0001) сапфировых подложках толщиной $0.43 \mathrm{~mm}$. Масс-спектрометрия вторичных ионов в выращенных образцах показала, что в качестве основных примесей присутствует кремний с $n_{\mathrm{Si}} \approx 1.5 \cdot 10^{20} \mathrm{~cm}^{-3}$, а также углерод и кислород, концентрации которых не превышают $\sim 5 \cdot 10^{18}$ и $\sim 10^{19} \mathrm{~cm}^{-3}$ соответственно. В качестве излучения накачки использовалась четвертая гармоника $\mathrm{Nd}$ :YAG-лазера с длиной волны $\lambda=266 \mathrm{~nm}$, длительностью импульса на полувысоте $8 \mathrm{~ns}$ и частотой следования $10 \mathrm{~Hz}$. Стимулированное излучение формировалось в результате усиления непрерывного пробного излучения в возбужденной области структуры. В качестве пробного выступало излучение от лампы накаливания, ограниченное спектральным фильтром в диапазоне $\lambda=350-700 \mathrm{~nm}$ и распространявшееся под углом $15^{\circ}$ к нормали структуры. Для измерения временно́го поведения излучение регистрировалось фотоумножителем в области 200-900 nm с временны́м разрешением $3 \mathrm{~ns}$ через спектральный фильтр - призменный монохроматор с полосой пропускания $10 \mathrm{~nm}$. Все эксперименты проводились при комнатной температуре. Подробное описание методики экспериментов представлено в [4,5].

На рис. 1 представлены экспериментальные временны́е зависимости затухания интенсивности люминесценции $I_{L}(t)$ структуры $\mathrm{Al}_{0.74} \mathrm{Ga}_{0.26} \mathrm{~N}$ на различных длинах волн излучения. Поведение этих кривых демонстрирует быструю (с экспоненциальным спадом за несколько 


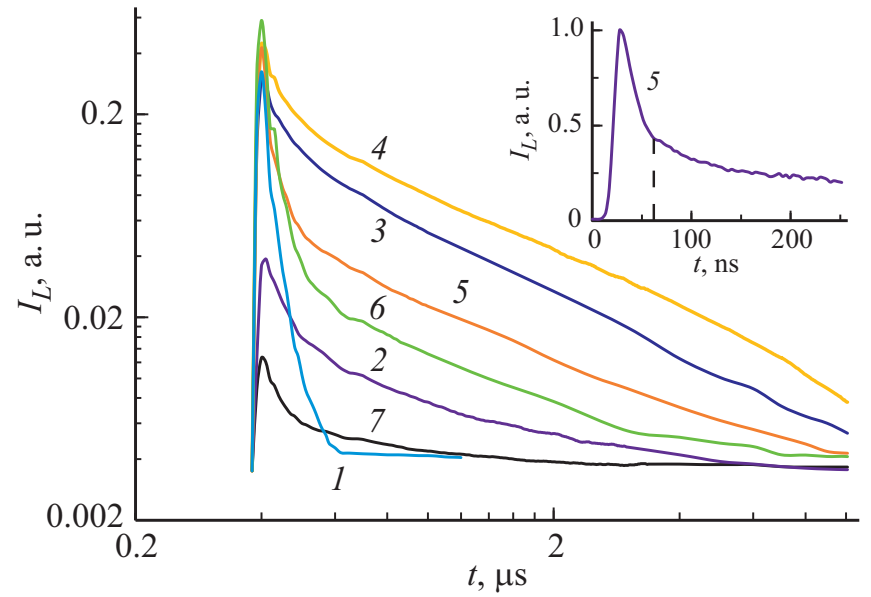

Рис. 1. Временны́е зависимости затухания интенсивностей люминесценции $I_{L}: \lambda=370$ (1), 380 (2), 420 (3), 480 (4), $520(5), 610(6)$ и $650 \mathrm{~nm}(7) . P_{p}=230 \mathrm{~kW} / \mathrm{cm}^{2}$. На вставке штриховой линией показана граница, разделяющая быструю и медленную компоненты излучения.

десятков наносекунд) и медленную (время затухания десятки микросекунд) компоненты. Штриховой линией на вставке к рис. 1 показана граница, находящаяся в точке перегиба кривых затухания, разделяющая быструю и медленную компоненты. Это подтверждают результаты аппроксимации экспериментальных данных суммой экспоненциальной и гиперболической функций. Быстрая компонента обусловлена электрон-акцепторной $(e-A)$ рекомбинацией, а медленная связана с рекомбинацией донорно-акцепторных пар (ДАП) [4,7]. В качестве донора выступает $\mathrm{Si}$, а в качестве акцептора может выступать катионная вакансия $\mathrm{Ga}$ и $\mathrm{Al}\left(V_{\mathrm{III}}\right)[8,9]$. Интенсивность стимулированного излучения $I_{S}(t)$ определялась исходя из экспериментальных данных по формуле

$$
I_{S}(t)=I_{L}^{*}(t)-I_{L}(t)
$$

где $I_{L}^{*}(t)$ - интенсивность люминесценции в присутствии пробного излучения с интенсивностью $I_{p r}$. Поскольку $I_{S}(t)$ прямо пропорциональна интенсивности $I_{p r}$, ее величина находилась из отношения $G_{S}(t)=I_{S}(t) / I_{p r}$, которое представляет собой оптическое усиление в возбужденной структуре. Полные интенсивности люминесценции $S_{L 0}$ и стимулированного излучения $S_{S 0}$ определялись как площади под кривыми $I_{L}(t)$ и $G_{S}(t)$ соответственно. Эти величины рассчитывались из экспериментальных данных по формулам

$$
S_{L 0}=\sum_{k=0}^{k=m} I_{L}\left(t_{k}\right) \Delta t, \quad S_{S 0}=\sum_{k=0}^{k=m} G_{S}\left(t_{k}\right) \Delta t .
$$

Диапазон времени вычисления от 0 до $t_{m} \approx 10-25 \mu \mathrm{s}$ устанавливался по моменту, когда убывающие по времени величины $I_{L}\left(t_{m}\right)$ и $G_{S}\left(t_{m}\right)$ становятся сравнимыми с шумами, а $\Delta t=t_{k+1}-t_{k}=1 \mathrm{~ns}$ - интервал между измеряемыми точками. По тем же формулам определялись интенсивности излучения для быстрых компонент люминесценции $S_{L F}$ и стимулированного излучения $S_{S F}$. В этом случае $t_{m}$ устанавливается по точкам перегиба на кривых $I_{L}(t)$ и $G_{S}(t)$ (вставка на рис. 1$)$.

На рис. 2, $a$ представлены спектры интенсивности $S_{L F}$, которые демонстрируют широкую полосу излучения $\Delta E \approx 1.6 \mathrm{eV}$, возникающую за счет процесса $e$ - $A$-рекомбинации с максимумами, сдвинутыми в длинноволновую область. Отношение интенсивности быстрой компоненты к полной интенсивности $S_{L F} / S_{L 0}$ имеет минимум в максимуме спектра излучения и возрастает на его краях. Спад интенсивности $S_{L 0}$ в длинноволновой части спектра связан с уменьшением вероятности рекомбинации ДАП, которая экспоненциально уменьшается с возрастанием расстояния $R$ между донором и акцептором. Рекомбинация ДАП в коротковолновой области обрывается, когда $R$ достигает минимального значения, равного периоду решетки.

Рис. 2, $b$ демонстрирует спектральные характеристики люминесценции и стимулированного излучения для

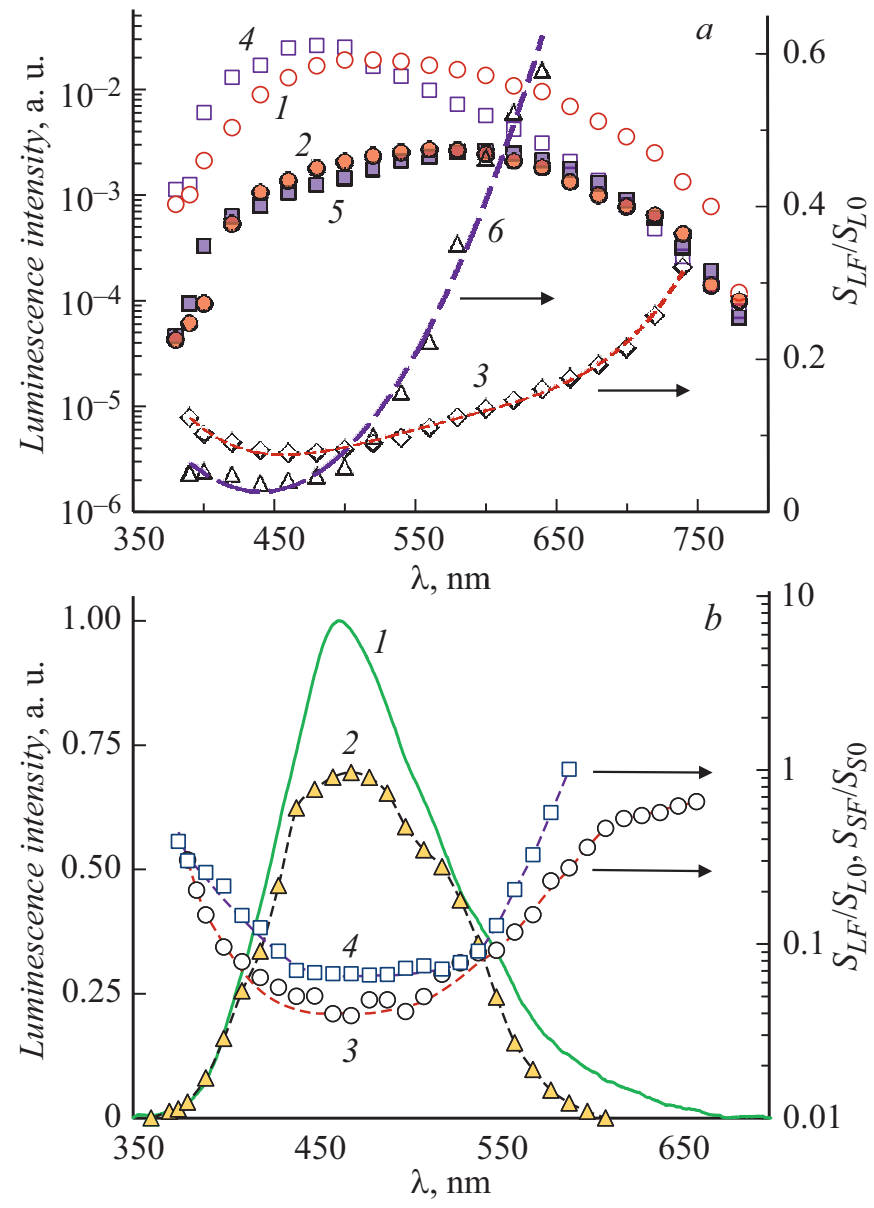

Рис. 2. Спектральные зависимости параметров. $a-$ структуры $\mathrm{Al}_{0.65} \mathrm{Ga}_{0.35} \mathrm{~N}(1-3)$ и $\mathrm{Al}_{0.74} \mathrm{Ga}_{0.26} \mathrm{~N}(4-6): 1,4-S_{L 0}$, $2,5-S_{L F}, 3,6-S_{L F} / S_{L 0} . P_{p}=52 \mathrm{~kW} / \mathrm{cm}^{2} . b-$ структура $\mathrm{Al}_{0.74} \mathrm{Ga}_{0.26} \mathrm{~N}: 1-S_{L 0}$ (с разрешением $\left.0.5 \mathrm{~nm}\right), 2-S_{S 0}, 3-$ $S_{L F} / S_{L 0}, 4-S_{S F} / S_{S 0} . P_{p}=230 \mathrm{~kW} / \mathrm{cm}^{2}$. 


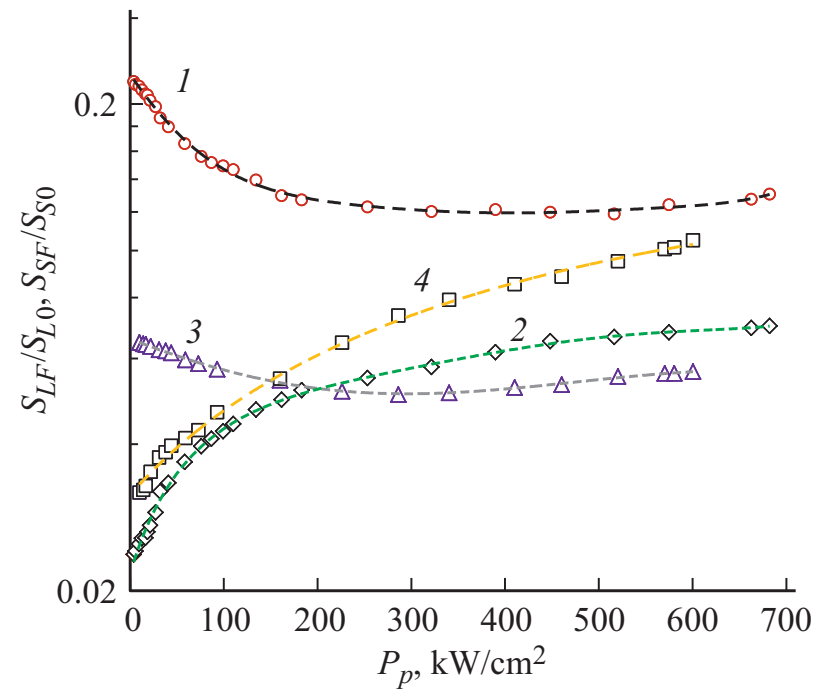

Рис. 3. Зависимости $S_{L F} / S_{L 0}\left(P_{p}\right)(1,3)$ и $S_{S F} / S_{S 0}\left(P_{p}\right)(2,4)$ для $\mathrm{Al}_{0.65} \mathrm{Ga}_{0.35} \mathrm{~N}(1,2)$ и $\mathrm{Al}_{0.74} \mathrm{Ga}_{0.26} \mathrm{~N}(3,4)$.

структуры $\mathrm{Al}_{0.74} \mathrm{Ga}_{0.26} \mathrm{~N}: \mathrm{Si}$. Спектральная зависимость для интенсивности стимулированной эмиссии $S_{S 0}$ достаточно хорошо совпадает со спектром люминесценции. Спектральные зависимости отношений для люминесценции $S_{L F} / S_{L 0}$ и стимулированной эмиссии $S_{S F} / S_{S 0}$ показывают значительный рост доли интенсивности быстрой компоненты люминесценции и стимулированного излучения на краях спектров излучения. Доля интенсивности $S_{S F}$ в центре контура излучения почти в 2 раза больше этой величины для люминесценции.

Экспериментальные результаты показывают, что интенсивности люминесценции $S_{L 0}$ и усиленного излучения $S_{S 0}$ при увеличении мощности накачки растут до $P_{p} \approx 100 \mathrm{~kW} / \mathrm{cm}^{2}$, а затем испытывают насыщение. Подобная картина поведения наблюдается и для интенсивностей быстрых компонент люминесценции $S_{L F}$ и стимулированной эмиссии $S_{S F}$.

Рис. 3 демонстрирует поведение отношений интенсивностей быстрых компонент люминесценции $S_{L F} / S_{L 0}$ и стимулированной эмиссии $S_{S F} / S_{S 0}$ к их полной интенсивности на длине волны, соответствующей центру люминесценции, в зависимости от плотности мощности накачки $P_{p}$. Доля быстрой компоненты люминесценции максимальна при малых уровнях накачки, затем уменьшается с насыщением при увеличении $P_{p}$. В то же время отношение $S_{S F} / S_{S 0}$ для интенсивности стимулированного излучения монотонно увеличивается с ростом $P_{p}$, а затем испытывает насыщение. Для $\mathrm{Al}_{0.74} \mathrm{Ga}_{0.26} \mathrm{~N}$ доля быстрой компоненты стимулированного излучения в центре контура излучения достигает $10 \%$.

Длительности импульсов быстрых компонент стимулированной эмиссии меньше в 1.3 раза относительно люминесценции и не зависят от мощности накачки. Для структуры $\mathrm{Al}_{0.65} \mathrm{Ga}_{0.35} \mathrm{~N}$ измеренная на полувысоте величина длительности импульса быстрой компоненты люминесценции равна $\sim 38 \mathrm{~ns}$, для $\mathrm{Al}_{0.74} \mathrm{Ga}_{0.26} \mathrm{~N} \sim 29 \mathrm{~ns}$. Усиление не зависит от ширины спектра пробного излучения. Широкий спектр излучения обеспечивает неоднородный характер уширения обоих процессов, ответственных за излучательную рекомбинацию. Рекомбинация ДАП содержит много перекрывающихся пиков, связанных с разными расстояниями ДАП. Ширина спектра для $e$-A-рекомбинации определяется широкой акцепторной полосой вследствие сильного легирования структур (см., например, $[4,5])$.

Представленные экспериментальные результаты демонстрируют свойства двух протекающих вместе процессов излучательной рекомбинации неравновесных носителей зарядов при импульсном оптическом возбуждении. Наибольший вклад в люминесценцию и стимулированную эмиссию в центре контура излучения вносит донорно-акцепторная рекомбинация с характерными временами релаксации до нескольких десятков микросекунд. Другой процесс $-e-A$-рекомбинация с временнб́м затуханием длительностью несколько десятков наносекунд имеет долю интенсивности излучения до $10 \%$.

Исследования особенностей процессов генерации излучения и оптического усиления в сильнолегированных структурах $\mathrm{Al}_{x} \mathrm{Ga}_{1-x} \mathrm{~N}: \mathrm{Si}$ могут привести к реализации новых элементов фотоники - активных сред с уникальными характеристиками.

\section{Финансирование работы}

Работа выполнена в рамках государственного задания ИФП СО РАН.

\section{Конфликт интересов}

Авторы заявляют, что у них нет конфликта интересов.

\section{Список литературы}

[1] D. Li, K. Jiang, X. Sun, C. Guo, Adv. Opt. Photon., 10 (1), 43 (2018). DOI: $10.1364 /$ AOP.10.000043

[2] K.A. Jones, T.P. Chow, T.P. Wraback, M. Shatalov, Z. Sitar, F. Shahedipour, K. Udwary, G.S. Tompa, J. Mater. Sci., 50 (9), 3267 (2015). DOI: 10.1007/s10853-015-8878-3

[3] Y. Nagasawa, A. Hirano, Appl. Sci., 8 (8), 1264 (2018). DOI: $10.3390 / a p p 8081264$

[4] P.A. Bokhan, N.V. Fateev, T.V. Malin, I.V. Osinnykh, Dm.E. Zakrevsky, K.S. Zhuravlev, J. Lumin., 203, 127 (2018). DOI: 10.1016/j.jlumin.2018.06.034

[5] П.А. Бохан, К.С. Журавлёв, Дм.Э. Закревский, Т.В. Малин, И.В. Осинных, Н.В. Фатеев, Письма в ЖТФ, 45 (18), 48 (2019). DOI: 10.21883/PJTF.2019.18.48239.17894

[6] P.A. Bokhan, N.V. Fateev, T.V. Malin, I.V. Osinnykh, Dm.E. Zakrevsky, K.S. Zhuravlev, Opt. Mater., 105, 109879 (2020). DOI: 10.1016/j.optmat.2020.109879 
[7] P.Y. Yu, M. Cardona, Fundamentals of semiconductors (Springer-Verlag, Berlin-Heidelberg, 2010), p. 345-426.

[8] Y. Taniyasu, M. Kasu, N. Kobayashi, Appl. Phys. Lett., 81 (7), 1255 (2002). DOI: 10.1063/1.1499738

[9] Q. Yan, A. Janotti, M. Scheffler, C.G. Van de Walle, Appl. Phys. Lett., 105 (1), 111104 (2014). DOI: 10.1063/1.4895786 